Sam R . K ottamasu L awrence R . Kuhns

\section{Musculoskeletal computed radiography in children: scatter reduction and improvement in bony trabecular sharpness using air gap placement of the imaging plate}

entrance radiation dose does not have to be increased for air gap digital radiography. The radiographic noise or quantum mottle is highest on the Bucky image, higher on air gap images and minimal on the table top images, despite a high scatter to primary radiation ratio at the table top. The lower quantum mottle on the table top images allowed for maximal visualization of low contrast densities in the soft tissues. Air gap radiography further improves musculoskeletal computed imaging by reducing the scatter to primary radiation ratio without an increase in the skin entrance dose. For significant reduction of the scatter to primary radiation ratio and best evaluation of line pair spatial resolution and bony trabeculae, a 25-in. (635-mm) air gap with digital radiography would be optimal. For evaluation of low contrast densities in the soft tissues, table top placement would be the technique of choice.
Department of Radiology,

Mott Children's Hospital,

University of Michigan, Ann Arbor,

Michigan, USA resolution were best on the 25 -in. (635-mm) air gap images. The skin

\section{Introduction}

We have used digital radiography for musculoskeletal imaging in the Pediatric Orthopedic Clinic at Children's Hospital of Michigan for 4 years and there has been good acceptance of the images by clinicians. During this time we have attempted to optimize the processing parameters of the Fuji Digital Radiography system (Fuji Medical Systems USA, Stamford, Conn.) for each age group and body part, using table top and Bucky (moving grid) techniques. We decided that the next step in improving the image quality would be to investigate the effect of various air gaps on scatter reduction, sharpness of bony trabeculae and line pair resolution, using a knee phantom to simulate an average skeletal part in children. 
Fig. 1 Scatter to primary radiation ratio $(\mathrm{S} / \mathrm{P}$ ratio $)$ at various air gaps below the knee phantom are displayed. S/P ratio decreased from a value of almost 1 at table top to 0.2 at 25 in. $(635-\mathrm{mm})$

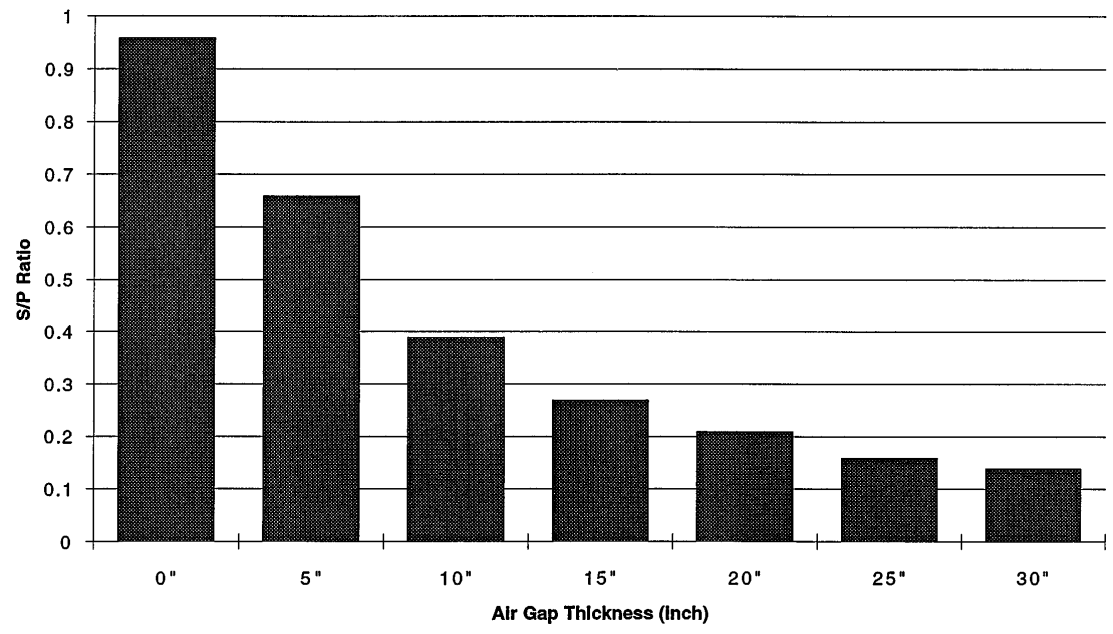

Table 1 Line pair resolution per $\mathrm{mm}$ at various positions of the phosphor plate

\begin{tabular}{lll}
\hline Receptor placement & Mean & $\begin{array}{l}\text { Standard } \\
\text { deviation }\end{array}$ \\
\hline Table top & 2.59 & 0.27 \\
Bucky & 2.79 & 0.44 \\
Air gap, 5 in. $(127 \mathrm{~mm})$ & 2.50 & 0.32 \\
Air gap, 10 in. $(254 \mathrm{~mm})$ & 2.78 & 0.17 \\
Air gap, 15 in. $(381 \mathrm{~mm})$ & 3.09 & 0.39 \\
Air gap, 20 in. $(508 \mathrm{~mm})$ & 3.20 & 0.33 \\
Air gap, 25 in. $(635 \mathrm{~mm})$ & 3.30 & 0.20 \\
Air gap, 30 in. $(762 \mathrm{~mm})$ & 3.20 & 0.33 \\
\hline
\end{tabular}

\section{Materials and methods}

A $0.3-\mathrm{mm}$ focal spot radiographic tube was placed 40 in. $(1016 \mathrm{~mm})$ above the table top. A standard knee phantom with a line pair phantom (Nuclear Associates, New York) taped to the under surface of the knee and acrylic beads of varying sizes from 1 to $5 \mathrm{~mm}$ in diameter taped over the soft tissue portion of the phantom was used. Fuji Standard III Imaging receptors were used and all images were processed on an AC-1 plus image processor using the same processing parameters (density, contrast, frequency filtering and edge enhancement). The images were obtained using Bucky placement, table top placement of the receptor, and placement of the receptor at 5, 10, 15, 20, 25, and 30 in. $(127,254,381,508,635$, and $762 \mathrm{~mm}$ ) below the table top after moving the table top aside. There was no modification of radiographic equipment. A table top technique of $65 \mathrm{kVp}$ and $3.2 \mathrm{mAs}$ was used for all images, including those obtained on the Bucky apparatus. The scatter to primary radiation ratio at various air gaps below the knee phantom was measured. These measurements were obtained using film densitometry and the beam stop method [1] by M. Goodsitt (Department of Radiology, University of Michigan). Film density measurements were translated into radiation exposure values using the characteristic curve (also called the $\mathrm{H}$ and $\mathrm{D}$ curve, after Hunter and Driffield, who first used such a curve) [2]. It was assumed that exposure levels in shadows of the beam stops represented only scattered radiation, while the levels immediately surrounding the beam stop represented scattered radiation plus unscattered primary radiation [1].
Two board-certified radiologists and a board-certified orthopedic surgeon independently evaluated all images without knowing the experimental conditions of the study. Each physician assessed sharpness of the bony trabeculae on each image on a 1-5 scale, with 5 being the best. They evaluated line pair resolution on all images using a $12 \times$ magnification loop. They then evaluated quantum mottle on each image on a 1-5 scale with 5 representing the greatest mottle. They next evaluated visualization of the low-contrast acrylic beads in the soft tissue portion of the phantom, with 1 being least and 5 being most visible.

\section{Results}

The scatter to primary radiation ratio decreased from a value of almost 1 at table top to about 0.4 at a 10 -in. (254-mm) air gap and about 0.2 at 25 in. (635-mm) (Fig.1). Line pair resolution per millimeter on Bucky and 10-inch (254-mm) air gap images was similar (Table 1). A progressive improvement in bony trabecular sharpness and line pair resolution, compared with the table top and Bucky images was noted on the air gap images from 10 through 25 in. (254-635-mm) (Figs. 2, 3, Table 1). Sharpness of the bony trabeculae and line pair resolution were best on the 25 -in. (635-mm) air gap images. The quantum mottle was highest on the Bucky image, higher on air gap images and least with the table top technique (Fig.4). Visualization of the low-contrast beads in the soft tissue area was best on the table top images (Fig.5). The 25-in. (635-mm) air gap images were next best for detection of the low-contrast beads. A combination of visualization of beads in the soft tissues and trabecular sharpness was best achieved on the 25in. (635-mm) air gap images (Fig. 6). 
Fig. 2 Trabecular detail. Scores for each observer and total scores for each imaging technique are shown. The lowest score was seen using the table-top technique, where the scatter to primary ratio is high. The highest scores were achieved at air gaps of 25 in. (635-mm) and 30 in. (762-mm), where the scatter was minimized

Fig. 3 Line pair resolution. The line pair resolution for each observer and the total line pair resolution for all three observers are displayed for each imaging technique. The line pair resolution was maximized using a 25-in. (635-mm) air gap
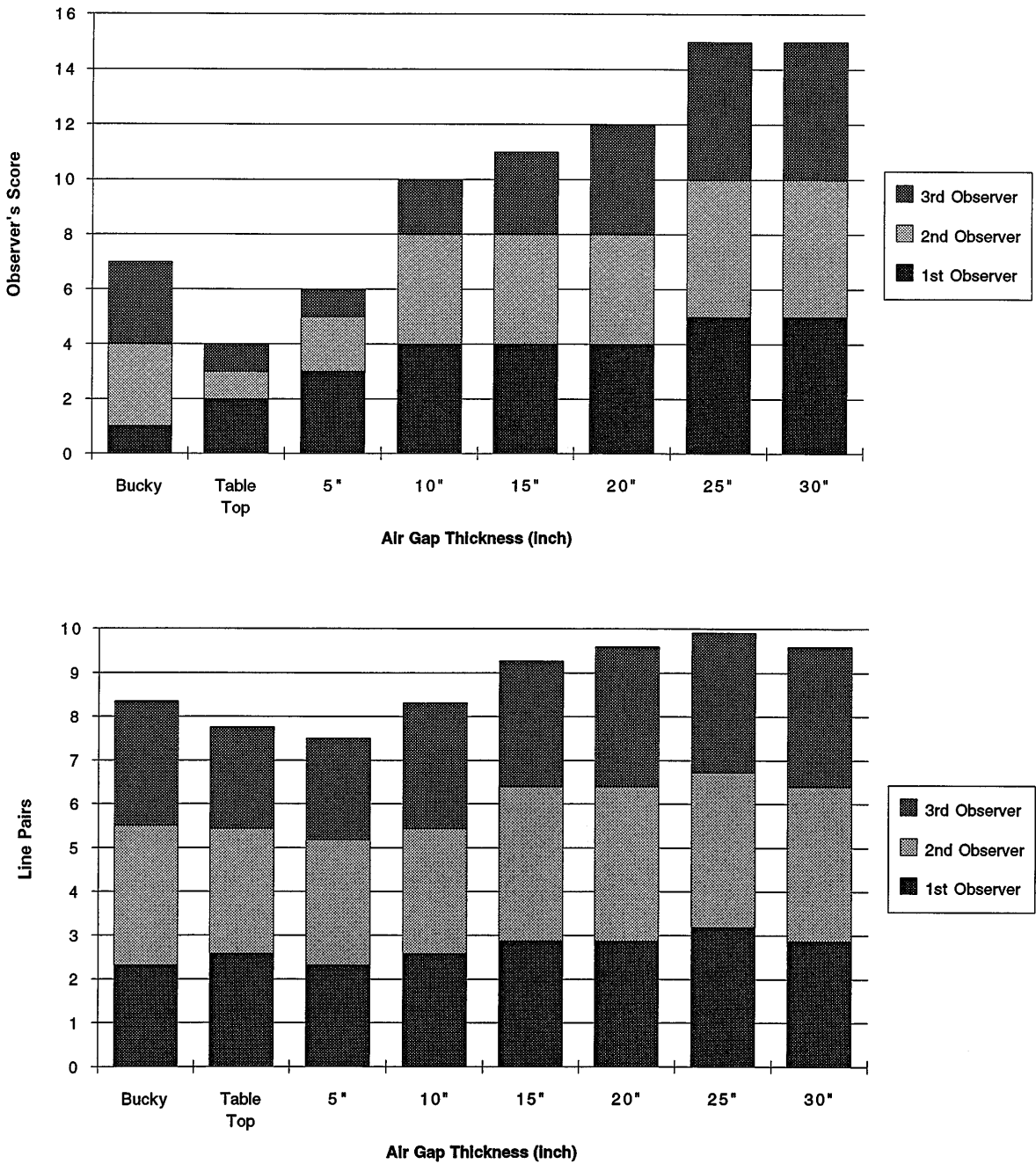

\section{Statistical analysis}

The inter-observer variability was evaluated using weighted Kappa analysis. The average Kappa value for all three paired observers was 0.73 for trabecular sharpness, 0.05 for beads in soft tissues, and 0.52 for quantum mottle. Values of weighted Kappa above 0.75 indicate excellent agreement beyond chance, whereas values below 0.40 indicate poor agreement [3]. Thus, trabecular sharpness alone was evaluated by our observers with fairly minimal inter-observer variability.

\section{Discussion}

Computed radiography (CR) is ideal for pediatric musculoskeletal imaging. Storage phosphor CR is a digitized imaging system, which uses conventional radiographic equipment to expose an image on a photostimulable imaging plate (IP) instead of a film-screen combination. Scatter reaching the IP is significantly less when a grid is employed. However, a grid absorbs about $40 \%$ of the total radiation emerging from the patient, which makes it necessary to increase the skin entrance dose [4].

The present project demonstrates that air gap radiography further improves musculoskeletal computed imaging by reducing the scatter to primary radiation ratio without an increase in the skin entrance dose. An air gap between the patient and the imaging plate reduces scatter reaching the receptor and also geometrically magnifies the object. Scatter to primary ratio is reduced significantly from a value of almost 1 at table top to about 0.4 at 10 -in. (254-mm) air gap and 0.2 at 25 -in. (635-mm) air gap placement of the IP (Fig. 1). Sharpness of the bony trabeculae and line pair resolution at 10-in. (254-mm) air gap are better than the table top images. For best evaluation of the sharpness of bony trabeculae and line pair resolution, a 25 -in. (635-mm) air gap would be optimal (Figs. 2, 3, 6). This represents only 1.6 times 
Fig. 4 Quantum mottle. The score of each observer for noise and the total score for all three observers is displayed for each imaging technique. The noise was greatest for the Buckey technique because of reduction of both scatter and primary radiation reaching the receptor. The noise was lowest for the table-top technique, where scatter radiation reaching the receptor was maximized
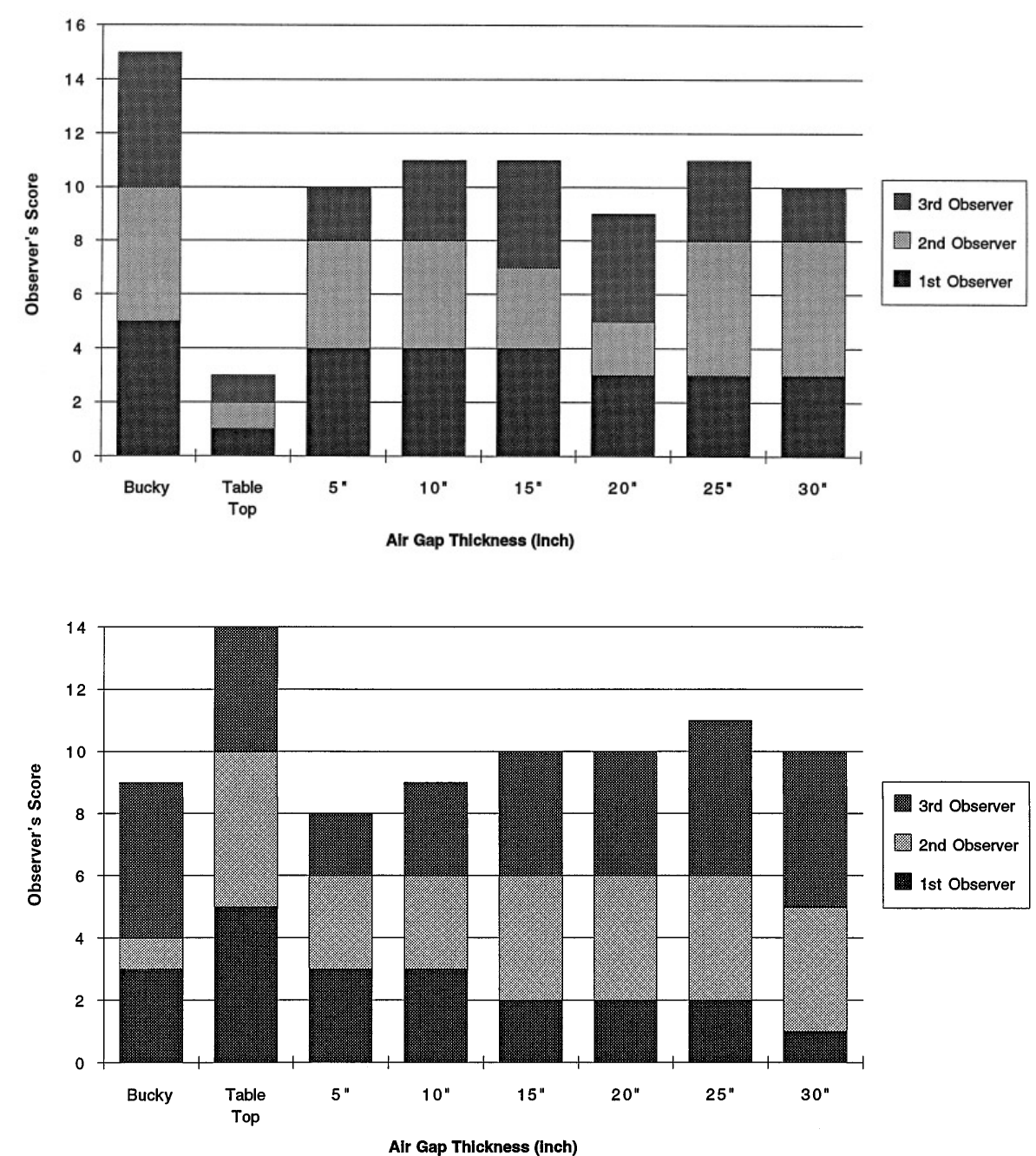

Air Gap Thickness (inch)
Fig. 5 Low-contrast objects (beads) in soft tissue. The scores of each observer and the total score for all three observers are displayed for each imaging technique. The visualization of the beads was best accomplished using the tabletop technique, where the total photon flux (scatter and primary radiation) was maximized geometric magnification. Standard reduction of a 14 in. $\times 17$ in. $(356-\mathrm{mm} \times 432-\mathrm{mm})$ image to a 10 in. $\times 12$ in. $(254-\mathrm{mm} \times 305-\mathrm{mm})$ hard copy by CR, therefore produces a near life-size image of the object, so that many body parts (elbow, knee, hand, wrist, shoulder) can be imaged using air gap placement of the IP.

The quantum mottle is inversely proportional to the total number of photons reaching the imaging plate. When the same exposure factors are used, the quantum mottle is highest on the Bucky image, higher on air gap images and minimal using the table top, non-grid technique (Fig. 4). When the air gap is used, more of the scattered photons do not reach the receptor and the total number of photons incident on the IP decreases resulting in greater quantum mottle. The lower quantum mottle allows maximal visualization of the low contrast densities in the soft tissues (Fig. 5) despite a high scatter to primary radiation ratio on the table top images (Fig.1). Thus for visualization of low-contrast foreign bodies in the soft tissues, table top placement of the receptor would be the chosen technique. For the best overall imaging technique for soft tissues and trabecular detail, a 25-in. (635-mm) air gap would be chosen (Fig. 6). Based on the above experiments, we have demonstrated that the same table top non-grid radiographic exposure technique can be used for air gap imaging with CR. The skin entrance dose does not have to be increased for air gap placement of the IP due to the linear response over a very wide dynamic range of the CR system $[5,6]$.

Murphey et al. [7] reviewed digitized radiographs of the original radiographs in 42 patients with non-displaced or minimally displaced fractures and found that all fractures were seen on digitized images with $2.88 \mathrm{LP} / \mathrm{mm}$ or greater resolution. Following a review of 122 digitized images of plain radiographs selected to represent a wide variety of musculoskeletal lesions, Wegryn [8] reported that $2.5 \mathrm{LP} / \mathrm{mm}$ was adequate to visualize all the abnormalities seen on plain radiographs. 

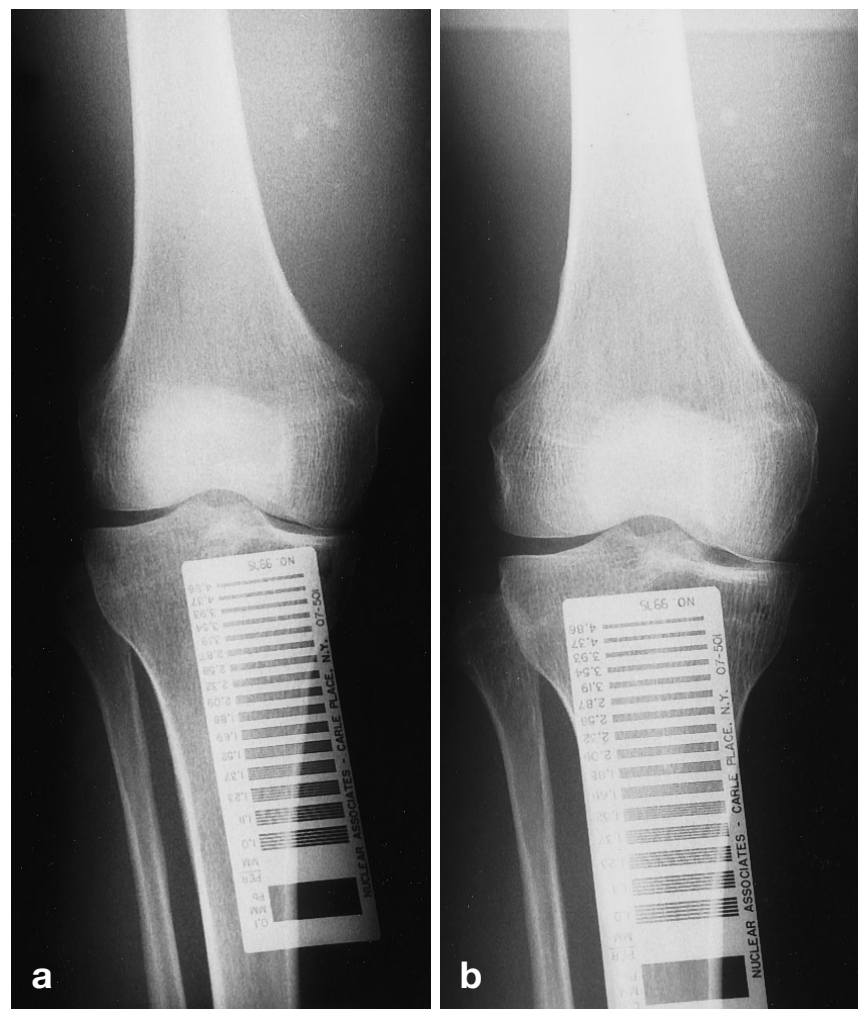

Fig. 6 a Table top and b 25-inch $(635-\mathrm{mm})$ air gap images of the knee with a line pair phantom. A marked improvement in bony trabecular sharpness and line pair resolution is noted on the 25inch $(635-\mathrm{mm})$ air gap image compared with the table top image
We know from our work compared with that of previous investigators that the line pair resolution achieved using an air gap of 10 in. (254-mm) or greater is more than adequate for routine diagnostic musculoskeletal imaging [6-12]. To date, we have performed air gap radiography in a few patients in the Pediatric Orthopedic Clinic for evaluation of the appendicular skeleton with good initial results. Modification of the radiographic tables used for acquiring CR images to include an adjustableheight cassette holder underneath a transparent table top for visualization of the field of collimation on the cassette would facilitate easy air gap placement of the IP for routine clinical use of this technique.

In conclusion, computed radiography is an alternative to film-screen radiography in the evaluation of the musculoskeletal system in children. A significant reduction in the scatter to primary radiation ratio, improvement in bony trabecular sharpness and line pair resolution can be achieved using air gap placement with $\mathrm{CR}$ without an increase in the skin entrance dose compared with the table-top technique.

A cknowledgements We thank Maureen A.Humphries for technical assistance, Drs. Thomas Slovis, Carl Stanitski, and Joseph Reed for acting as independent observers in this study, Mitch Goodsitt, $\mathrm{PhD}$ for measurement of scatter to primary radiation and Anthony Schork, $\mathrm{PhD}$ for performing the statistical analysis. Statistical support was provided by the University of Michigan Multipurpose Arthritis Center, NIH grant P60-AR220557-12.

\section{References}

1. Sorenson JA, Nelson JA, Niklason LT, Jacobsen SC (1980) Rotating disk device for slit radiography of the chest. Radiology 134: 227-231

2. Michel M, Ter-Pogossian (1969) The physical aspects of diagnostic radiology, Harper \& Row, New York

3. Landes JR, Koch GG (1974) The measurement of observer agreement for categorical data. Biometrics 33: 159174

4. Tucker DM, Souto M, Barnes GT (1993) Scatter in computed radiography. Radiology 188: 271-274

5. Kuhns LR, Kottamasu SR (1995) Pediatric air-gap chest digital imaging: an experimental study. Pediatr Radiol 25: S99-S201
6. Neitzel U (1992) Grids or air gaps for scatter reduction in digital radiography: a model calculation. Med Phys 19: 475481

7. Murphey MD, Bramble JM, Cook LT, Martin NL, Dwyer SJ (1990) Nondisplaced fractures: spatial resolution requirements for detection with digital skeletal imaging. Radiology 174: 865870

8. Wegryn SA (1990) Comparison of digital and conventional musculoskeletal radiography: an observer performance study. Radiology 175: 223-228

9. Murphey MD (1989) Digital skeletal radiography: spatial resolution requirements for detection of subperiosteal resorption. AJR 152: 541-546
10. Richmond BJ, Powers C, Piraino DW, Freed H, Meziane MA, Hale JC, Schluchter MD, Schils J, Gragg LA (1992) Diagnostic efficacy of digitized images vs. plain films: a study of the joints of the fingers. AJR 158: 437-441

11. Wilson AJ (1991) Photostimulable phosphor digital radiography of the extremities: diagnostic accuracy compared with conventional radiography. AJR 157: 533-538

12. Murphey MD, Quale JL, Martin NL, Bramble JM, Cook LT, Dwyer SJ III (1992) Computed radiography in musculoskeletal imaging: state of the art. AJR 158: 1071-1080 